\title{
Polyarginine decorated Polydopamine nanoparticles with antimicrobial properties for functionalisation of hydrogels
}

\author{
Céline Muller ${ }^{1}$, Emine Berber ${ }^{1}$, Gaetan Lutzweiler ${ }^{1}$, Ovidiu Ersen ${ }^{2}$, Mounib Bahri², $^{2}$ \\ Philippe Lavalle ${ }^{1}$, Vincent Ball ${ }^{1}$, Nihal Vrana ${ }^{1}$, and Julien BARTHES ${ }^{1}$ \\ ${ }^{1}$ INSERM UMR-S 1121 \\ ${ }^{2}$ IPCMS, Insititut de Physique et de Chimie des Matériaux de Strasbourg
}

May 5, 2020

\begin{abstract}
Polydopamine (PDA) nanoparticles are versatile structures that can be stabilized with proteiins.In this study, we have demonstrated the feasibility of developping PDA/polypeptides complexe in the shape of nanoparticles. The polypeptide can also renders the nanoparticle functional. Herein, we have developed antimicrobial nanoparticles with a narrow size distribution by decorating the polydopamine particles with a chain-length controlled antimicrobial agent Polyarginine (PAR). The obtained particles were $3.9 \pm 1.7 \mathrm{~nm}$ in diameter and were not cytotoxic at 1:20 dilution and above. PAR-decorated nanoparticles have demonstrated a strong antimicrobial activity against S. aureus, one of the most common pathogen involved in implant infections. The minimum inhibitory concentration is 5 times less than the cytotoxicity levels. PAR-decorated nanoparticles are incorporated into gelatin hydrogels used as a model of tissue engineering scaffolds and to confer them strong antimicrobial properties without affecting their stability and biocompatibility while improving their mechanical properties (increased storage modulus). Decorated Polydopamine particles can be a versatile tool for functionalization of hydrogels in regenerative medicine applications by providing bioactive properties.
\end{abstract}

\section{Introduction}

Tissue engineering and regenerative medicine use biomaterials, cells and bioactive agents to engineer implantable structures to replace damaged tissues and organs. In many cases, the size of the tissue to be replaced is considerable and once in the body, the engineered tissue might have immune privileged zones separated from the host circulation, which can create local havens for bacterial attachment and biofilm formation. This constitutes a similar situation to that of peri-implantitis (infections around dental implants) which can affect 5 to more than $10 \%$ of dental implants.(Koldsland, Scheie, \& Aass, 2010) As tissue engineering solutions become more and more common, there is a requirement for inclusion of antimicrobial agents as a safety precaution. However, such antimicrobial agents should not trigger the development of antimicrobial resistance or any undesired side effects and preferably they should have additional properties that can support the main function of the scaffold. One of the potential ways of achieving such an effect is the inclusion of nanoparticles functionalised with antimicrobial agents in scaffolds.

Polydopamine (PDA) films are versatile coatings obtained through the oxidation of dopamine(H. Lee, Dellatore, Miller, \& Messersmith, 2007) or other catecholamines(Kang, Rho, Choi, Messersmith, \& Lee, 2009) using either dissolved oxygen in basic solutions or other strong oxidants.(Ponzio et al., 2016; Wei, Zhang, Li, Li, \& Zhao, 2010) Knowing that PDA displays many structural similarities with eumelanin,(Meredith \& Sarna, 2006) the black-brown pigment of the skin, and that eumelanin grains display a structural hierarchy,(Clancy \& Simon, 2001) motivating us to explore specific controlled ways to synthesize PDA to obtain 
well defined and stable nanoparticles. Since eumelanin grains of the skin are always surrounded by proteins,(Mani, Sharma, Tamboli, \& Raman, 2001) the natural way to control the size of PDA particles is to add proteins(Chassepot \& Ball, 2014) or other polymers(Arzillo et al., 2012; Mateescu, Metz-Boutigue, Bertani, $\&$ Ball, 2016) to the dopamine solution during its oxidation. The properties of transferrin capped PDA nanoparticles(Hauser et al., 2018) and the photothermal properties of such nanoparticles(Han et al., 2016) have already been explored. These inherent properties in term of photothermal sensitivity and versatile surface chemistry have shown great potential for biomedical applications. For example, PDA NPs photothermal properties have been used recently to develop drug release carriers for cancer therapy.(Poinard et al., 2018; Wang et al., 2019) Moreover, the versatile surface chemistry of PDA NPs have allowed the conjugation of various bioactive agents (proteins, polyelectrolytes) though different mechanisms such as $\pi-\pi$ stacking, electrostatic interactions and nucleophile addition of amine to quinone (Michael addition).(Ball, 2018) The possible functionalization of the PDA nanomaterials combined with their low toxicity made them good candidates to develop bioactive carriers with properties relevant for biomedical purposes such as anti-cancer, pro-angiogenic, anti-inflammatory or antimicrobial activities.(Chen et al., 2015; Chia-Che Ho, 2013; S. Li et al., 2019)

We have recently demonstrated that chain-length controlled polyarginine (PAR) can be used as an antimicrobial agent inside thin films without having any adverse effect on mammalian cell behaviour at concentrations several times higher than its minimal inhibitory concentration.(Knopf-Marques et al., 2019; Mutschler et al., 2016) The polycationic nature of polyarginine is the underlying reason for such antimicrobial activity, as evidenced by the high frequency of arginine residues in naturally occurring antimicrobial peptides. Moreover, polyarginine has been shown to have cell penetration properties and has been utilised for DNA and RNA delivery.(Hu, Lou, \& Wu, 2019; Kim, Davaa, Myung, \& Park, 2010) Thus, incorporation of polyarginine in tissue engineering scaffolds such as hydrogels would provide not only the required antimicrobial properties, but it can also be used for gene delivery within the scaffold to enhance their bioactive properties. Hence PAR can be potentially used for decoration of PDA nanoparticles while rendering them antimicrobial and by guaranteeing that the decoration does not hinder its antimicrobial activity.

Hydrogels due to their intrinsic properties in term of high-water absorption, viscoelastic properties and biocompatibility can closely simulate properties of living tissues. In particular hydrogels made of natural polymer-based components such as collagen, gelatin, chitosan or alginate are of particular interest since they can mimic the natural microenvironment of an extracellular matrix.(Hoffman, 2012; K. Y. Lee \& Mooney, 2001) For this reason, these materials represent good candidates for biomedical applications such as drug delivery, wound dressing or tissue engineering.(Hamedi, Moradi, Hudson, \& Tonelli, 2018; Hunt, Chen, van Veen, \& Bryan, 2014; J. Li \& Mooney, 2016; Mohamad, Loh, Fauzi, Ng, \& Mohd Amin, 2019; Zilberman, 2015) Nevertheless, their use is still limited by their poor mechanical properties and their lack of stability in physiological condition. To overcome these limitations, multiple strategies have been proposed to reinforce hydrogels. These strategies include i) the crosslinking of the hydrogel either chemically (creation of covalent bonds between macromolecular chains), physically (entanglement, ionic bonds, H-bonds) or biologically (nucleotide pairing, self-assembly, enzymatic crosslinking)(Place, George, Williams, \& Stevens, 2009) or ii) the use of filler agents such as nanoparticles or nanofibers.(Sahraro, Barikani, \& Daemi, 2018; Sheffield, Meyers, Johnson, \& Rajachar, 2018; Zhou \& Wu, 2011) With both strategies the mechanical behaviour of the hydrogel could be improved but with the use of fillers, the hydrogel's bio-functionality can also be tuned by the intrinsic properties of nanoparticles (such as silver NP which are antimicrobial)(Ribeiro et al., 2017) or by functionalizing these nanoparticles with bioactive molecules. Thus, in this context, PAR decorated PDA particles can be used to render hydrogels antimicrobial and change their mechanical properties simultaneously.

The risk of contamination is a serious problem in implantable devices as infection can even lead to implant failure and in this aspect, most of the hydrogels used in the biomedical field are also concerned by this issue.(Salomé Veiga \& Schneider, 2013) As a consequence, antimicrobial strategies must be envisioned in all biomedical products development.(Ng et al., 2014; Ribeiro et al., 2017; Yu et al., 2007) PDA based nanoparticles have been previously utilized to elaborate, biocatalysts,(El Yakhlifi, Ihiawakrim, Ersen, \& Ball, 
2018) cell-targeting agents(W.-Q. Li et al., 2017) or theranostic agent(Dong et al., 2016) but to our knowledge, protein capped PDA nanoparticles have never been used for antimicrobial applications particularly in conjugation with hydrogels. Hence, we aim to develop a gelatin based hydrogel with antimicrobial properties and enhanced mechanical properties through the loading of polydopamine nanoparticles decorated with an antimicrobial agent, polyarginine. This gelatin based hydrogel should provide the optimal microenvironment for cell encapsulation while PAR decorated PDA particles will prevent bacterial contamination after implantation. To achieve this, the following hypotheses must be validated:

i) PDA NPs should demonstrate the feasibility of being decorated with PAR,

ii) the immobilization of PAR at the surface of PDA NPs should not interfere with antimicrobial properties of PAR,

iii) PAR decorated PDA particles should retain their antimicrobial properties in gelatin hydrogels without affecting the biocompatibility of the hydrogels.

2. Materials and Methods

2.1 Materials

Dopamine hydrochloride ( $\mathrm{Mw}=189,64$ Da, H8502, CAS: 62-31-7), Tris(hydroxymethyl) aminomethane $(\mathrm{Mw}=121.1 \mathrm{Da}, \mathrm{T}-1503$, CAS: $77-86-1)$ and Gelatin Type A from porcine skin $\left(\mathrm{Mw}=5-10 \times 10^{4} \mathrm{Da}\right)$ were purchased from Sigma-Aldrich (St Quentin Fallavier, France). Poly(L-arginine hydrochloride) with 30 arginine residues $\left(\mathrm{PAR}_{30}, \mathrm{Mw}=5.8 \mathrm{kDa}\right.$, CAS: 26982-20-7) was purchased from Alamanda Polymers (US). Microbial-Transglutaminase (Activa, 86-135 units/g) was kindly provided by Ajinomoto (Japan).

2.2 Methods

2.2.1 Synthesis of polydopamine nanoparticles (PDA NPs) and PDA NPs decorated with PAR 30 (PDA$\mathrm{PAR}_{30}$ )

$\mathrm{PAR}_{30}$ powder was dissolved in TRIS buffer $50 \mathrm{mM}$ at $\mathrm{pH}=8.5$ at a concentration of $1 \mathrm{mg} \cdot \mathrm{mL}^{-1}$. Then the $\mathrm{PAR}_{30}$ solution was mixed with dopamine powder in order to obtain a concentration of $0.2 \mathrm{mg} \cdot \mathrm{mL}^{-1}$. This solution was stirred for 24 hours under stirring (300 RPM) (Scheme 1). After that, 7 steps of dialysis in $1000 \mathrm{~mL}$ TRIS buffer were performed to remove the PAR 30 non deposited onto PDA NPs. Final solution was stored at $+4^{\circ} \mathrm{C}$. PDA NPs were synthesized using the same protocol just by removing $\mathrm{PAR}_{30}$ deposition step.

\subsubsection{Synthesis Fluorescently labeled PAR 30 -FITC NPs}

PAR30 chains were labeled with fluorescein isothiocyanate (FITC, Sigma-Aldrich, France) using a protocol previously described by Mutschler et al.(Mutschler et al., 2016)

\subsubsection{Dynamic Light Scattering and Zeta Potential Measurements}

The hydrodynamic diameter of the PDA-PAR 30 particles was measured by dynamic light scattering (Malvern Nano ZS) in a backscattering configuration $\left(\vartheta=173^{\circ}\right)$ and at a wavelength of $632.8 \mathrm{~nm}$ (HeNe laser). The intensity autocorrelation function obtained from a $1 \mathrm{~mL}$ suspension (after dialysis) was analyzed with the Contin algorithm. The volume distribution as a function of the hydrodynamic diameter was obtained thereof using a refractive index of $1.73 \pm 0.02 \mathrm{i}$ at the wavelength of the experiment. At least three measurements were realized for each sample.

Zeta potential measurements were performed using Nano-ZS device (Malvern Instruments, UK). At least three measurements were realized for each sample in order to compare zeta potential between PDA NPs and PDA-PAR 30 NPs.

2.2.4 Elaboration of Gelatin hydrogel loaded with PDA-PAR ${ }_{30}$ NPs (Gel-PDA-PAR) 
Gelatin hydrogel was prepared with gelatin type A at $6 \% \mathrm{w} / \mathrm{v}$ in PBS as control (Gel-PBS). As PDA-PAR 30 NPs were prepared in Tris buffer, Gel-PDA-PAR 30 composite hydrogel was prepared in mix PBS/Tris at different ratios. For example, Gel-PDA-PAR ${ }_{30}$ is prepared by adding directly PDA-PAR ${ }_{30}$ NPs in Tris buffer solution to gelatin type A powder. In this condition PDA-PAR $\mathrm{P}_{30}$ NPs were non diluted in the hydrogel. Then Gel-PDA-PAR 30 1:2 is prepared by first diluting PDA-PAR 30 NPs in Tris buffer solution with PBS at a ratio 1:1 (Tris/PBS) (V/V) and then dissolving gelatin powder in this solution. The same method is used to prepare the other formulations. Then Gelatin solution is heated at $+40-50^{\circ} \mathrm{C}$ until complete solubilization. After that, microbial- Transglutaminase (TGA, enzymatic crosslinker) prepared in PBS at $20 \% \mathrm{w} / \mathrm{v}$ is added in a ratio 1 to $5(200 \mu \mathrm{L}$ of TGA for $1000 \mu \mathrm{L}$ of gelatin). After a mixing step, $200 \mu \mathrm{L}$ gelatin solution are poured into molds or 48 wells-plate. This crosslinking step is performed overnight at $+4^{\circ} \mathrm{C}$. Finally, hydrogels are treated with UV light for 15 minutes before being stored at $4^{\circ} \mathrm{C}$ prior to use.

\subsubsection{Microscopy analyses}

\section{Scanning Electron Microscope (SEM)}

NPs or Gel-NPs samples were let to dry overnight under a fume hood prior to be observed with a SEM Hitachi SU8010 microscope under 1KeV voltage acceleration. Low-angle Back-scattered electron (LA-BSE) detector has been used to acquire both information on the composition of the sample, and on topography.

\section{Cryo-Transmission Electron Microscope}

For the cryo-TEM characterization a drop of the same PDA-PAR 30 particle suspension was deposited on a carbon coated electron transmission microscopy grid. The drop was aspired with filter paper to obtain a particle containing film covering the whole carbon membrane. Those coated grids were immersed in ethane at the temperature of liquid nitrogen, transferred on the cryo-holder and inserted in the electron microscope. Analysis was performed at $200 \mathrm{kV}$ with a Jeol $2100 \mathrm{~F}$ transmission electron microscope. To reduce the irradiation damage, the images were acquired using a low-density electron beam.

\section{Epifluorescent Microscope}

Fluorescence pictures were acquired with the Nikon Eclipse Ti-S microscope with x10 objective equipped with Nikon Digital camera (DS-Q11MC, NIS-Elements software, Nikon, France) and processed with ImageJ (https://imagej.nih.gov/ij/).

\subsubsection{Rheology}

Rheological properties were measured using a Kinexus Ultra + rheometer (Malvern Panalytical, UK). For the determination of the shear viscosity and viscoelastic properties $\left(\mathrm{G}^{\prime}\right)$, we used a plate geometry of $20 \mathrm{~mm}$ diameter, a gap of $0.5 \mathrm{~mm}$ and the temperature was kept constant at $37^{\circ} \mathrm{C}$. Shear viscosity measurement were carried out with a shear rate ranging from $10^{-1}$ to $10^{3} \mathrm{~s}^{-1}$. Gelatin and Gel-PDA-PAR 30 1:1 (non-diluted) were prepared as previously described without microbial-TGA and then $200 \mu \mathrm{L}$ of these solutions were put in the rheometer at $37^{\circ} \mathrm{C}$. Viscoelastic properties measurement were performed with a frequency ranging from 0.1 to $50 \mathrm{~Hz}$ at a fixed strain of $0.5 \%$. For this experiment, microbial-Transglutaminase was added to Gelatin and Gel-PDA-PAR 30 solutions at a ratio 1:5 and then $200 \mu \mathrm{L}$ of these solutions were put in the rheometer at $37^{\circ} \mathrm{C}$ and let crosslinked overnight before performing the sweep frequency experiment.

\subsubsection{Stability (enzymatic, $p H 5$ and $P B S$ )}

To study stability of our gelatin hydrogel containing PDA-PAR 30 NPs (Gel-PDA-PAR 30 1:1) non-dilute we used three different testing configurations: i) stability in physiological $\mathrm{pH}$, ii) in acidic condition and iii) in the presence of enzyme (collagenase).

For the experiment at physiological pH, we used PBS solution. Hydrogel samples were placed in 24 wells-plate with $1 \mathrm{~mL}$ of PBS $1 \mathrm{X}$ from Dutscher, at $+37^{\circ} \mathrm{C}$. Measurements were carried out after vacuum-drying step for 4 hours then we weighed samples. Finally, we put them in a new PBS solution and put them back at $+37^{\circ} \mathrm{C}$ 
until next measurement. We performed these measurements at different time point: initial measurement (before stability experiment) then at days 1, 4, 7 and 15 .

For the experiment in acidic condition $(\mathrm{pH}=5)$, protocol was the same as the previous one with the same follow-up at different time point. We used a citrate-phosphate buffer $(0.15 \mathrm{M}, \mathrm{pH}=5)$.

To test enzymatic stability, we used collagenase (Sigma-Aldrich) solution at $0.1 \% \mathrm{w} / \mathrm{v}$. Measurements were performed using the same protocol but with different time points. Measurements were realized at t0, 30 minutes, 1 hour and 2 hours. All samples were washed three times with PBS 1X in order to remove collagenase enzyme, dried with paper and stock until next day to perform vacuum-drying and weigh. All results were compared to gelatin hydrogel without NPs condition. All samples were performed in triplicates.

\subsubsection{Swelling}

To study swelling ratio of our gelatin hydrogel containing PDA-PAR 30 NPs non-diluted (Gel-PDA-PAR 30 ). We performed a first vacuum-dry step to obtain initial weigh. Then, samples were immersed in PBS 1X at $+37^{\circ} \mathrm{C}$. Measurements were performed after drying briefly with a paper. We realized these measurements at different time points: 5, 10, 20, 30, 60, 120, 180 and 240 minutes. All results were compared to gelatin hydrogel without NPs condition. All samples were performed in triplicates.

\subsubsection{Biological assays}

\section{Cytotoxicity assay}

Cytotoxicity test is adapted from ISO STANDARD procedure 10993-5. Balb 3 T3 clone A31 cell line (mouse fibroblast) was purchased from ATCC (CCL-163). The culture medium used was DMEM High Glucose with stable glutamine with pyruvate sodium (L0103-500) supplemented with $10 \%$ v/v of Foetal Bovine Serum (S1810-500) from Dutscher and 1\% v/v penicillin-streptomycin (15140-122) from Gibco. Preculture was performed in 24 wells-plate in order to obtain $80 \%$ confluency after 24 hours. Two ways for cytotoxicity were studied for our samples. First, PDA-PAR 30 NPs were added in solution at different dilutions onto cell layer (direct assay) for 24 hours. We realized a PDA-PAR 30 dilution of 1:10 in cell culture medium (ratio 1 to 9 Tris/Cell culture medium) to keep a normal cells viability, so the minimal dilution tested is 1:10. Then to increase the dilution of PDA-PAR $\mathrm{P}_{30} \mathrm{NPs}$, we first diluted in Tris Buffer and then keep the same ratio of 1 to 9 Tris/Cell culture medium. Secondly, we tested gelatin hydrogels with PDA-PAR ${ }_{30}$ NPs at different dilutions, they were incubated in cell medium for 24 hours in order to test release from hydrogels, in parallel of cells preculture. Gel-PDA-PAR 30 were prepared as previously explained. These vehicles extract was added onto cells layer (extract assay) for 24 hours. All volumes were fixed at $1 \mathrm{~mL}$. After exposure of samples onto cell layer (extract and direct assays) we performed MTT test. We also took bright field pictures at x10 before and after samples exposition to observe cells morphology. We compared all results to a growth control condition (cells cultured in cell culture medium without contact with hydrogel or NPs) defined as $100 \%$ viability. We also tested Tris buffer (in solution or in the hydrogel) as a negative control to check its non-cytotoxicity. We used sodium azide 3M (in solution or loaded in the hydrogel) as positive control. All samples were performed in triplicates.

\section{Antibacterial assay}

Staphylococcus aureus strain is purchased from ATCC (25923).S. aureus was cultured at $+37^{\circ} \mathrm{C}$ in a Mueller Hinton Agar (BD) for 24 hours. Then on colony was transferred to $10 \mathrm{~mL}$ of Mueller Hinton Broth (BD) for 24 hours at $+37^{\circ} \mathrm{C}$ with agitation for preculture step. The absorbance is read at $620 \mathrm{~nm}$ and we adjusted DO value to 0.001 to perform our assay, corresponding to a density of $8.10^{5} \mathrm{CFU} \cdot \mathrm{mL}^{-1}$. As cytotoxicity assay, we tested PDA-PAR 30 NPs in solution and also Gel- PDA-PAR 30 composite hydrogel. For solution part, we kept the same ratio (samples:bacteria) in medium as 1 to 9 , final volume is $100 \mu \mathrm{L}$ (10 $\mu \mathrm{L}$ of NPs for $90 \mu \mathrm{L}$ of bacteria in medium). Samples with bacteria were incubated for 24 hours at $+37^{\circ} \mathrm{C}$ with agitation. Final absorbance were read at $620 \mathrm{~nm}$ in order to quantify bacteria growth or inhibition. For gelatin hydrogels part, samples were directly produced in 48 wells-plate and sterilized by UV light exposure. After preculture of S. aureus, we added $300 \mu \mathrm{L}$ of suspension with $\mathrm{OD}=0.001$ then we incubated for 24 hours at $+37^{\circ} \mathrm{C}$ 
with agitation. OD was read at $620 \mathrm{~nm}$. We compared PDA NPs and PDA-PAR 30 NPs. In solution, OD of samples (PDA and PDA-PAR ${ }_{30}$ ) with bacteria were subtracted to OD the same samples without bacteria to remove PDA absorption (brown solution). All results were compared to a normal growth condition named "Bacteria", defined as $100 \%$ growth. We also tested Tris buffer (in solution or hydrogel) as a negative control. We used antibiotics (Cefotaxime $0.1 \mu \mathrm{g} \cdot \mathrm{mL}^{-1}+$ Tetracycline $10 \mu \mathrm{g} \cdot \mathrm{mL}^{-1}$ ) as positive control. We also used $\mathrm{PAR}_{30}\left(10 \mu \mathrm{g} . \mathrm{mL}^{-1}\right)$ without PDA as positive control. All samples were performed in triplicates.

\section{Bacteria Live Dead Assay}

We performed an additional test after antibacterial assay with gelatin hydrogels to evaluate the amount of bacteria alive or dead on the surface of samples. We used the BacLight Redox Sensor CTC Vitality Kit (Molecular Probes). SYTO 24 green-fluorescent nucleic acid staining is used at $0.001 \mathrm{mM}$ to counting all bacteria and CTC red staining is used at $50 \mathrm{mM}$ to detect bacteria alive.

\section{Results}

\subsection{Synthesis and characterization of PDA-PAR 30 NPs}

In this study, polydopamine nanoparticles (PDA NPs) were synthesized using a protocol developed by Chassepot et al.(Chassepot \& Ball, 2014) In this work, the ability of proteins (either negatively or positively charged) to stabilize and control the size of dopamine aggregates formed in dopamine solutions upon oxidation in order to get functional nanoparticles was demonstrated. In the current work, we have used a chainlength controlled polycation, polyarginine with 30 residues $\left(\mathrm{PAR}_{30}\right)$, known to have a strong antimicrobial activity.(Mutschler et al., 2016) Then we have synthesized PDA-PAR P $_{30}$ nanoparticles by adding a PAR $_{30}$ solution (1 mg. $\left.\mathrm{mL}^{-1}\right)$ in contact with different concentrations of dopamine hydrochloride solution ranging from 0.2mg. $\mathrm{mL}^{-1}$ to $0.5 \mathrm{mg} \cdot \mathrm{mL}^{-1}$ in alkaline Tris Buffer $(\mathrm{pH}=8.5)$ for 24 hours under stirring (Scheme 1). We first studied the influence of the dopamine concentration on the nanoparticle stability. After 2 months in a closed vial, only the formulation synthesized with $0.2 \mathrm{mg} / \mathrm{mL}$ of dopamine hydrochloride (PDA-PAR 30 0.2 ) was still stable without apparent sedimentation (Figure 1A). For the other formulations containing 0.5 and $0.3 \mathrm{mg} \cdot \mathrm{mL}^{-1}$ of dopamine hydrochloride (noted as PDA-PAR 30 0.5 and PDA-PAR 30 0.3) a total sedimentation was observed after few days. The size of the aggregates was analysed using dynamic light scattering (Figure S1). Aggregates at the microscale were found for both formulations. The size of these aggregates was estimated at about $2 \mu \mathrm{m}$ and $4 \mu \mathrm{m}$ for the PDA-PAR 300.5 and PDA-PAR 300.3 formulations

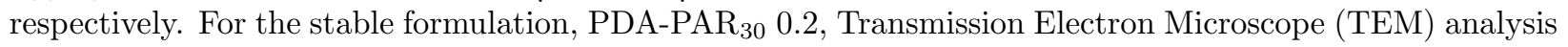
was performed to determine the size of the resulting nanoparticles (Figure 1B). Nanoparticles with a narrow size distribution of $3.9 \pm 1.7 \mathrm{~nm}$ (mean diameter based on the measurement of 300 nanoparticles) were obtained. As a control, we also synthesized PDA NPs using the same protocol but without any decoration but these particles were not stable and formed aggregates between 1-10um (Figure S1). The only way to obtain stable PDA based NPs was to use polypeptides or proteins to stabilize them. Thus, for the rest of the experimental work, we used the stable nanoparticles obtained with a solution of $0.2 \mathrm{mg}$. $\mathrm{mL}^{-1}$ of dopamine hydrochloride (PDA-PAR 30.2 condition). To further probe and demonstrate the decoration of polydopamine NPs with chain-length controlled polyarginine, zeta potential measurements were performed. It is known that polydopamine is negatively charged above $\mathrm{pH} 5$. Indeed, the measurement on PDA NPs gave a zeta potential value of $-22.7 \mathrm{mV}$ (Figure $1 \mathrm{C}$ ). By adding PAR 30 to the PDA particles, we obtained zeta potential value of $+30.3 \mathrm{mV}$. The surface charge of the NPs has switched from negative to positive values as $\mathrm{PAR}_{30}$ is a polycation. This demonstrates that the polycation was successfully deposited on the surface of PDA NPs.

In the next step, we have investigated the antimicrobial properties of PDA-PAR ${ }_{30} 0.2$ NPs to check if the PAR chains maintained their biological activity once immobilized at the surface of PDA NPs. Indeed, our previous studies demonstrated the need for the mobility of PAR molecules in the coatings to exert their antimicrobial activity and this mobility in the particles is not guaranteed.(Mutschler et al., 2016) We have tested different NPs dilutions ranging from 1:10 to 1:100 from the initial particle concentration and incubated them with $S$. aureus $\left(8.10^{5}\right.$ CFU.mL ${ }^{-1}$ ) for 24 hours. As a control, we also synthesized unmodified PDA 
NPs and used them in the same range of dilution against $S$. aureus. The results are presented in Figure 2A. PDA-PAR $_{30} 0.2$ NPs ranging from 1:10 to 1:50 in dilution completely inhibit the bacterial growth (100\% growth inhibition) and then from the dilution $1: 100$, we can again observe a significant bacterial growth (40\% of growth inhibition for the dilution 1:100). Based on these results, we can deduce that the MIC (Minimum inhibitory concentration) of these PDA-PAR 30 0.2 NPs is between 1:50 and 1:100. As it was difficult to estimate the true concentration of NPs, based on the absence of a precise knowledge about the dopamine oxidation-oligomerization reaction yield, we have performed this experiment on different batches of NPs coming from different synthesis done in identical conditions. The MIC was always estimated between 1:50 and 1:100 (Figure S2) which means that the synthesis is reproducible, and we can assume that we always obtain comparable amount of NPs of similar size in each synthesis. As a control, experiments performed on PDA NPs without the decoration of $\mathrm{PAR}_{30}$ show absence of antimicrobial activity whatever the dilution used. This means that the antimicrobial activity of the NPs can be attributed to the presence of PAR $\mathrm{R}_{30}$ and that $\mathrm{PAR}_{30}$ chains maintain their biological activity after immobilization on PDA NPs.

After the evidence of the antimicrobial properties of the NPs, it was necessary to check their cytotoxicity. For this purpose, we used Balb 3T3 cells as a well-established model (mouse fibroblasts). PDA-PAR 300.2 NPs at different dilutions in cell culture medium were put in contact with an almost confluent layer of 3T3 cells for 24 hours and the viability of these cells was then estimated with a MTT assay and compared to a control (normal medium without NPs). A material is defined as cytotoxic if a decrease of at least $30 \%$ in viability compared to the control is observed according to ISO/EN 109935. The obtained results are presented in Figure 2B and images of the cell morphology are given in Figure S3. As a control, we also investigated the cytotoxicity of Tris Buffer at a dilution $1: 10$ which represents the highest concentration of Tris used in the experiments to dilute NPs in the cell culture medium. At this concentration of Tris Buffer, it was shown that the buffer was not cytotoxic. This indicates that for the rest of the experiment, if a cytotoxic effect is monitored, it can only be attributed to the presence of PDA-PAR nanoparticles. Finally, it was demonstrated that for PDA-PAR 30 0.2 NPs, only the 1:10 dilution exhibits a cytotoxic behaviour with a decrease in viability of about $90 \%$. Hence, we can conclude that PDA-PAR 30 0.2 NPs particles are non-cytotoxic at dilutions higher than 1:10.

3.2 Design and characterization of antimicrobial Gelatin hydrogels loaded with PDA-PAR ${ }_{30}$ NPs (Gel-PDA$\left.\mathrm{PAR}_{30}\right)$

We have previously demonstrated that decoration of PDA NPs with $\mathrm{PAR}_{30}$ allows to confer antimicrobial properties. In the second part of the study, we have incorporated these PDA decorated NPs into gelatin hydrogels to develop an antimicrobial hydrogel that can be further used as an implant surface coating to fight infection after implantation while providing a degradable environment for remodelling. For this purpose, PDA-PAR 30 0.2 NPs were incorporated into gelatin type A hydrogel without any dilution (GelPDA-PAR 30 and the hydrogels were then crosslinked with microbial-Transglutaminase in order to reinforce their mechanical properties and stability. This hydrogel Gel-PDA-PAR ${ }_{30} 1: 1$ is depicted in Figure $3 \mathrm{~A}$ and compared to gelatin hydrogel without NPs (Gel). We observed a brown color for the hydrogel loaded with NPs which is the characteristic colour of PDA. The presence of NPs at the surface of the gelatin hydrogel was checked using SEM and it was found that the particles were homogeneously distributed. However, we can notice that once incorporated in the hydrogel, there is an increase of particle size from few nanometers (as found for solution dispersed NPs, Figure 1B) to almost $100 \mathrm{~nm}$. This can be explained by the aggregation of the NPs during the hydrogel formation (Figure 3B). We then investigated the swelling properties of the composite hydrogels and compared them to pure gelatin hydrogels (Figure 3C). It has been found that the addition of NPs in the gelatin network had an effect on the swelling behaviour: a significant increase with a value of $500 \%$ was monitored, compared to pure gelatin hydrogel where a value of only about $300 \%$ was measured. This could be explained by Donnan effect due to the high positive charge density of the PAR covered NPs inducing a strong incorporation of counteranions and hence of hydration water.(Rathna, Mohan Rao, \& Chatterji, 1996) Then, the release of PDA-PAR 30 0.2 NPs from the gelatin hydrogel was studied using fluorescently labelled NPs with PAR $_{30}$-FITC (Figure 3D). We can observe a burst release of the NPs during the first hours with most of the NPs released during the first 3 days. However, the hydrogel was still 
brown after 3 days of release (data not shown), which means that some of the NPs were still present in the gelatin network even if no further release was recorded.

Next, we investigated the rheological properties of the gelatin-PDA-PAR 30 hydrogel composite to determine the influence of the addition of PDA-PAR 30 NPs on the mechanical properties. We first analysed the shear viscosity in solution of the two formulations ( $\mathrm{Gel}$ and Gel-PDA-PAR $\mathrm{P}_{30}$ ) without the crosslinking agent, microbial Transglutaminase (Figure 4A). The shear viscosity of both formulations remained constant over a large range of shear rates (from 0.1 to $1000 \mathrm{~s}^{-1}$ ). Moreover, the addition of nanoparticles in the formulation did not affect the shear viscosity since the values of both Gel and Gel-PDA-PAR 30 formulations were similar with values ranging from 0.1 to 0.06 Pa.s depending on the shear rate applied. After demonstrating that the addition of NPs in the formulation did not affect their viscosity, we crosslinked both formulations with microbial-transglutaminase and studied the viscoelastic properties of the resulting hydrogels. The two hydrogels were crosslinked for at least 16 hours in situ in the rheometer to ensure complete crosslinking of the network. Then we recorded the storage modulus of the two hydrogels as a function of frequency (Figure $4 \mathrm{~B})$. Over the frequency range tested $(0.1-50 \mathrm{~Hz})$, it was found that the storage modulus (G') of the two formulations increased slightly as a function of frequency. In addition, we observed a higher storage modulus in the case of Gel-PDA-PAR 30 hydrogel composite with values between 8 to almost $11 \mathrm{kPa}$, whereas for the gelatin hydrogel the values were between 5 and $7 \mathrm{kPa}$. Thus, the addition of NPs within PDA-PARhydrogel enhanced its viscoelastic properties. We can assume that the NPs act as a filler in the structure of the hydrogel.

After characterization of the mechanical properties of the hydrogels, we performed a stability study in different conditions (Figure 4C, D, E): enzymatic stability in the presence of collagenase, stability in acidic conditions $(\mathrm{pH}=5)$ as a model of infection and stability in physiological $\mathrm{pH}$ at 7.4 in PBS. All these experiments were conducted at $37^{\circ} \mathrm{C}$ and the mass of hydrogels were recorded as a function of time. For enzymatic stability, hydrogels were incubated in $1 \mathrm{~mL}$ of collagenase solution $\left(1 \mathrm{mg} \cdot \mathrm{mL}^{-1}\right)$ at $37^{\circ} \mathrm{C}$. In Figure $4 \mathrm{C}$, it is shown that the two formulations, Gel or Gel-PDA-PAR30, were completely degraded after 2 hours with the same kinetics. For the stability at $\mathrm{pH}=5$, hydrogels were incubated in $1 \mathrm{~mL}$ of citric buffer and the experiment was performed for 7 days with an estimation of mass loss at days 1, 4 and 7 (Figure 4D). After the first day, the hydrogel containing PDA-PAR ${ }_{30}$ NPs showed better stability ( $86 \%$ of remaining hydrogel versus 69 $\%$ for the pure gelatin hydrogel). But during the next days, the degradation was indistinguishable for both formulations. After 7 days, the difference between the two hydrogels was not significant (64\% degradation for Gel-PDA-PAR 30 vs $59 \%$ for Gel). The last stability experiment was also conducted for 7 days in PBS buffer at $\mathrm{pH}=7.4$ (Figure $4 \mathrm{E}$ ) and the results showed the same degradation profile between the two formulations with values of remaining hydrogel after 7 days of about $55 \%$ ( $56 \%$ for Gel-PDA-PAR 30 vs $53 \%$ for Gel). With this set of experiments, we can conclude that the addition of PDA-PAR ${ }_{30}$ NPs in the gelatin structure only increases the viscoelastic properties of the hydrogel (increase of G', Figure 4B) but did not change the stability of the resulting hydrogels compared to pure gelatin hydrogels.

Once the hydrogel composites were characterized, their antimicrobial activity starting from a dilution 1:1 (no dilution) and until $1: 50$ was tested against S. aureus (Figure 5A). Hydrogels were prepared in PBS but as the NPs were prepared in Tris Buffer, the final hydrogel composite was prepared in a PBS/Tris mix. It was found that Gel-PDA-PAR 30 hydrogels exhibit complete antimicrobial activity with a $100 \%$ growth inhibition after 24 hours in contact withS. aureus until a dilution of 1:4. For higher dilutions (1:10 and 1:50), the antimicrobial activity was lost because the NPs were probably too diluted in the hydrogel to maintain a biological activity. SEM analyses were also performed to compare the behaviour of Gel-PDA-PAR 30 (1:4) vs Gel (Figure 5B). After 24 hours of contact with $S$. aureus, the bacteria fully colonized gelatin hydrogel and started to form a biofilm whereas with the NPs loaded hydrogel almost no bacteria were found. Zooming on the Gel-PDA-PAR 30 surface allows to show the presence of particles at the surface of the hydrogel which confer the antimicrobial activity.

These results are also in agreement with the metabolic activity of bacteria, using CTC/Syto 24 staining where no viable bacteria were found on the hydrogel loaded with NPs (Figure S4). For final applications, 
the gel formulation with NPs diluted in a ration 1:4 seems to be optimal. Then, the cytotoxicity of the formulation Gel-PDA-PAR 30 . has been tested with Balb 3T3 cells using extraction method following ISO Standard 10993-5 recommendations. As the hydrogel composite was prepared in a PBS/Tris mix, we have first tested the cytotoxicity of pure gelatin hydrogels prepared in either Tris or PBS and then the cytotoxicity of hydrogel prepared in different ratios of PBS/Tris (Figure S5). It was found that none of the configurations tested exhibited cytotoxicity with viability up to $70 \%$ maintained in all cases. Then, we prepared Gel-PDA$\mathrm{PAR}_{30}$ hydrogel composites starting from a dilution $1: 4$ and until 1:50 (NPs were also diluted from 1:4 to 1:50 in the hydrogel) and studied their cytotoxicity. None of the formulations tested were cytotoxic and a viability up to $85 \%$ was maintained (Figure 6). The resulting cell morphology is given in Figure S6 and there was no evidence of anormal cell shape. The formulation 1:4 was tested towards Balb 3T3 cells and its viability was estimated to be around $90 \%$. Hence we can conclude that this formulation was not cytotoxic. Thus, we have designed a powerful antimicrobial hydrogel ony based on natural and bioderived materials, i.e gelatin, polydopamine and polyarginine. An adequate formulation has been obtained both in term of antimicrobial activity with $100 \%$ bacteria growth inhibition and in term of biocompatibility.

\section{Discussion}

4.1 Polyarginine decorated PDA NPs are stable and antimicrobial

Polydopamine NPs-based chemistry is versatile and should open the road to develop bioactive biomaterials with the possibility to decorate nanoparticles with multiple bioactive agents enabling us the design of hydrogel composites with bioactive properties. As these particles have a wide range of inherent properties as a potential substrate for photothermal effect or as quenchers and catalysts, they can be used in many biomedical applications including cancer therapy and stimuli responsive drug delivery. Adding a layer of bioactive agents on this structure creates a tool that is highly modular and can have applications in incorporating theranostic delivery and biosensing for tissue engineering and regenerative medicine applications. In this study, our main goal was to create stable polydopamine based nanoparticles with antimicrobial properties and to do that we have used a known polypeptide antibiotics (polyarginine).

In our study, we have first demonstrated that the addition of a sufficient amount of $\mathrm{PAR}_{30}$ to a sufficiently diluted solution of dopamine, $0.2 \mathrm{mg} \cdot \mathrm{mL}^{-1}$, in Tris buffer allows to produce small PDA-PAR and stable nanoparticles having an average diameter of $3.9 \pm 1.7 \mathrm{~nm}$ and displaying a positive zeta potential of about $+30.3 \mathrm{mV}$ compared to pristine PDA nanoparticles having a zeta potential close to $-23 \mathrm{mV}$ in the same conditions (Figure 1D).

Polyarginine, a positively charged polymer, has been originally used as a highly efficient cell penetrating peptide for gene delivery applications. Beyond this cell penetrating properties, due to its polycationic nature, polyarginine is a widely used component to build polyelectrolyte multilayers. Recently, it has also been shown that $\mathrm{PAR}_{18}$ and also its D-enantiomer has neuroprotective properties.(Meloni et al., 2019) As mentioned before, polyarginine also has antimicrobial activity whose efficacy is related to the mobility of PAR chains within polyelectrolyte structures.(Dong et al., 2016) In solution, a wide range of polyarginine chain lengths have shown antimicrobial activity; however in film configurations where polyarginine was in interaction with polyanions (heparin, chondroitin sulfate, alginate, hyaluronic acid) only a narrow range of chain length and only films with HA were active. Thus, decoration of polyarginine on PDA particles might have hindered the antimicrobial activity of polyarginine due to the lack of mobility.

However, our experiments demonstrated that polyarginine chains on PDA surfaces sustain their antimicrobial capacity. Indeed, the PDA-PAR 30 0.2 NPs display an antimicrobial activity against $S$. aureus up to a dilution of about 1:50 to 1:100 (Figure 2A) whereas the PDA NPs are not antimicrobial whatever their dilution; this demonstrates that the antimicrobial activity arises from the presence of PAR on its surface and also that the particle formation does not hinder the antimicrobial activity of PAR, excepting the cases of thin layerby-layer coatings where the antimicrobial properties depend on the polyanion. This is due to the fact that, even though immobilised, PAR chains are at the surface of the particles thus they are free to electrostatically interact with bacterial membranes even though the interaction is partially shared with the interaction with 
the PDA surface. Our experimental results clearly demonstrated that such interactions cannot overshadow the antimicrobial properties of polyarginine.

4.2 PDA-PAR NPs incorporated hydrogels have improved viscoelastic properties and antimicrobial capacity

The need for antimicrobial coatings for non-degradable implants has been long recognized such with systems such as TYRX ${ }^{\mathrm{TM}}$ antimicrobial, degradable polymer pouch used for pacemakers in clinical applications. The surface of non-degradable implants, can create an immune-privileged zone that can facilitate the bacterial attachment and the biofilm formation. For example, the rate of infections with fixation devices were estimated between 2-5\% The replacement of passive implants with engineered artificial tissues can in one way alleviate this problem as the porous nature of such scaffolds will enable the angiogenesis. Consequently, the implanted structure will integrate with the host immune system rather than being an isolated surface. But, on the other hand, materials such as natural polymers (as for instance collagen, gelatin, hyaluronic acid etc., with certain exceptions like chitosan) used for scaffolds generation can also boost the attachment and proliferation of bacteria just as well that of the host tissue. Moreover, potential use of engineered tissues in non-sterile wounds would also necessitate an antimicrobial component. Previously, such precautions has been tried to be put in place by either doping hydroxyapatite with silver or copper to confer antimicrobial activity to it(Sahithi et al., 2010; Stanić et al., 2011) or incorporation of silver based nanoparticles in the scaffold formulations. (Saravanan et al., 2011) Specifically, with gelatin foams crosslinked with genipin, Yazdimamaghani et al. demonstrated antimicrobial activity against $E$. coli and $S$. aureus by in situ formed silver nanoparticles.(Yazdimamaghani et al., 2014) However, it has been shown that silver nanoparticles are less cytotoxic compared to silver sulfadazine which at high concentrations can demonstrate hepatotoxicity in clinical settings.(Trop et al., 2006) Moreover, at the cellular level, it has been shown that silver exhibits mild cytotoxicity against mesenchymal stem cells, which are one of the major cell sources in tissue engineering.(Samberg, Loboa, Oldenburg, \& Monteiro-Riviere, 2012) Thus, alternative antimicrobial solution would be beneficial, particularly in formats that would also contribute to the mechanical properties of the overall scaffold in tissue engineering. The nanoparticles developed in this study could potentially achieve these two effects concomitantly in the context of hydrogels.

In this study, to demonstrate such utility of PDA-PAR NPs, we have used gelatin hydrogels, which creates a highly amenable surface for bacterial attachment as demonstrated by our results. The incorporation of the particles in the gel structure resulted in an increase in G' values which indicates a slightly stiffer hydrogel due to the nanoparticle based reinforcement. The incorporation of the particles in the gel structures could decrease their mobility and as a result their antimicrobial capacity; however, experiments showed that it was possible to maintain antimicrobial activity of the NPs at dilutions below the cytotoxic limits, the reason for this observation being the ability of the hydrogels to release the NPs as demonstrated by the release experiments. The relatively controlled manner of release of the NPs also points out using the hydrogels as a potential delivery system for such NPs.

\section{Conclusion}

Herein, we demonstrated that PDA based nanoparticles with a controlled size can be obtained in the presence of a polycationic antimicrobial peptide without interfering with the antimicrobial properties of the polypeptide. The system was effective not only in stand-alone configuration but also within hydrogels. The incorporation of the resulting NPs in gelatin hydrogels as a model of tissue engineering scaffolds leads to hydrogels with antimicrobial capacity and improved mechanical properties. These NPs can be used to render tissue engineering scaffold antimicrobial; particularly in the cases where the slow kinetic in the cell growth can result in bacterial pouches while contributing to the mechanical properties of the scaffold. Last but not least, we showed that this hydrogel can release NPs which would also allow to use it for drug release applications

Our future work will focus on the possibility to enable the muco penetration of the polyarginine decorated PDA nanoparticles.

\section{Supporting Information}


Supporting Information is available from the Wiley Online Library or from the author.

Conflicts of interest

"There are no conflicts to declare".

Acknowledgements

This project has received funding from the European Union's Horizon 2020 research and innovation programme under grant agreement No 760921(PANBioRA).

References

Arzillo, M., Mangiapia, G., Pezzella, A., Heenan, R. K., Radulescu, A., Paduano, L., \& d'Ischia, M. (2012). Eumelanin Buildup on the Nanoscale: Aggregate Growth/Assembly and Visible Absorption Development in Biomimetic 5,6-Dihydroxyindole Polymerization. Biomacromolecules, 13 (8), 2379-2390. doi:10.1021/bm3006159

Ball, V. (2018). Polydopamine Nanomaterials: Recent Advances in Synthesis Methods and Applications. Frontiers in Bioengineering and Biotechnology, 6 (109). doi:10.3389/fbioe.2018.00109

Chassepot, A., \& Ball, V. (2014). Human serum albumin and other proteins as templating agents for the synthesis of nanosized dopamine-eumelanin. Journal of Colloid and Interface Science, 414, 97-102. doi:https://doi.org/10.1016/j.jcis.2013.10.002

Chen, X., Huang, Y., Yang, G., Li, J., Wang, T., H. Schulz, O., \& K. Jennings, L. (2015). Polydopamine Integrated Nanomaterials and Their Biomedical Applications. Current Pharmaceutical Design, 21 (29), $4262-4275$.

Chia-Che Ho, S.-J. D. (2013). The pH-controlled nanoparticles size of polydopamine for anticancer drug delivery. Journal of Materials Science: Materials in Medicine, 24 (10), 2381-2390. doi:https://doi.org/10.1007/s10856-013-4994-2

Clancy, C. M. R., \& Simon, J. D. (2001). Ultrastructural Organization of Eumelanin from Sepia officinalis Measured by Atomic Force Microscopy.Biochemistry, 40 (44), 13353-13360. doi:10.1021/bi010786t

Dong, Z., Gong, H., Gao, M., Zhu, W., Sun, X., Feng, L., . . . Liu, Z. (2016). Polydopamine Nanoparticles as a Versatile Molecular Loading Platform to Enable Imaging-guided Cancer Combination Therapy. Theranostics, 6 (7), 1031-1042. doi:10.7150/thno.14431

El Yakhlifi, S., Ihiawakrim, D., Ersen, O., \& Ball, V. (2018). Enzymatically Active Polydopamine @ Alkaline Phosphatase Nanoparticles Produced by NaIO4 Oxidation of Dopamine. Biomimetics, 3 (4), 36.

Hamedi, H., Moradi, S., Hudson, S. M., \& Tonelli, A. E. (2018). Chitosan based hydrogels and their applications for drug delivery in wound dressings: A review. Carbohydrate Polymers, 199 , 445-460. doi:https://doi.org/10.1016/j.carbpol.2018.06.114

Han, L., Zhang, Y., Lu, X., Wang, K., Wang, Z., \& Zhang, H. (2016). Polydopamine Nanoparticles Modulating Stimuli-Responsive PNIPAM Hydrogels with Cell/Tissue Adhesiveness. ACS Applied Materials $\mathcal{E}^{3}$ Interfaces, 8 (42), 29088-29100. doi:10.1021/acsami.6b11043

Hauser, D., Estermann, M., Milosevic, A., Steinmetz, L., Vanhecke, D., Septiadi, D., . . . Rothen-Rutishauser, B. (2018). Polydopamine/Transferrin Hybrid Nanoparticles for Targeted CellKilling.Nanomaterials, 8 (12), 1065.

Hoffman, A. S. (2012). Hydrogels for biomedical applications.Advanced Drug Delivery Reviews, 64, 18-23. doi:https://doi.org/10.1016/j.addr.2012.09.010

Hu, J., Lou, Y., \& Wu, F. (2019). Improved Intracellular Delivery of Polyarginine Peptides with Cargoes. The Journal of Physical Chemistry B, 123 (12), 2636-2644. doi:10.1021/acs.jpcb.8b10483 
Hunt, J. A., Chen, R., van Veen, T., \& Bryan, N. (2014). Hydrogels for tissue engineering and regenerative medicine. Journal of Materials Chemistry B, 2 (33), 5319-5338. doi:10.1039/C4TB00775A

Kang, S. M., Rho, J., Choi, I. S., Messersmith, P. B., \& Lee, H. (2009). Norepinephrine: MaterialIndependent, Multifunctional Surface Modification Reagent. Journal of the American Chemical Society, 131 (37), 13224-13225. doi:10.1021/ja905183k

Kim, H.-K., Davaa, E., Myung, C.-S., \& Park, J.-S. (2010). Enhanced siRNA delivery using cationic liposomes with new polyarginine-conjugated PEG-lipid. International Journal of Pharmaceutics, 392 (1), 141-147. doi:https://doi.org/10.1016/j.ijpharm.2010.03.047

Knopf-Marques, H., Barthes, J., Lachaal, S., Mutschler, A., Muller, C., Dufour, F., . . . Vrana, N. E. (2019). Multifunctional polymeric implant coatings based on gelatin, hyaluronic acid derivative and chain length-controlled poly(arginine). Materials Science and Engineering: C, 104 , 109898. doi:https://doi.org/10.1016/j.msec.2019.109898

Koldsland, O. C., Scheie, A. A., \& Aass, A. M. (2010). Prevalence of Peri-Implantitis Related to Severity of the Disease With Different Degrees of Bone Loss. Journal of Periodontology, 81 (2), 231-238. doi:10.1902/jop.2009.090269

Lee, H., Dellatore, S. M., Miller, W. M., \& Messersmith, P. B. (2007). Mussel-Inspired Surface Chemistry for Multifunctional Coatings.Science, 318 (5849), 426-430. doi:10.1126/science.1147241

Lee, K. Y., \& Mooney, D. J. (2001). Hydrogels for Tissue Engineering. Chemical Reviews, 101 (7), 1869-1880. doi:10.1021/cr000108x

Li, J., \& Mooney, D. J. (2016). Designing hydrogels for controlled drug delivery. Nature Reviews Materials, 1 , 16071. doi:10.1038/natrevmats.2016.71

https://www.nature.com/articles/natrevmats201671\#supplementary-information

Li, S., Zhang, L., Liang, X., Wang, T., Chen, X., Liu, C., . . . Wang, C. (2019). Tailored synthesis of hollow $\mathrm{MOF} /$ polydopamine Janus nanoparticles for synergistic multi-drug chemo-photothermal therapy. Chemical Engineering Journal, 378 , 122175. doi:https://doi.org/10.1016/j.cej.2019.122175

Li, W.-Q., Wang, Z., Hao, S., He, H., Wan, Y., Zhu, C., . . . Zheng, S.-Y. (2017). Mitochondria-Targeting Polydopamine Nanoparticles To Deliver Doxorubicin for Overcoming Drug Resistance. ACS Applied Materials $\&$ Interfaces, 9 (20), 16793-16802. doi:10.1021/acsami.7b01540

Mani, I., Sharma, V., Tamboli, I., \& Raman, G. (2001). Interaction of Melanin with Proteins - The Importance of an Acidic Intramelanosomal pH. Pigment Cell Research, 14 (3), 170-179. doi:doi:10.1034/j.16000749.2001.140306.x

Mateescu, M., Metz-Boutigue, M.-H., Bertani, P., \& Ball, V. (2016). Polyelectrolytes to produce nanosized polydopamine. Journal of Colloid and Interface Science, 469 , 184-190. doi:https://doi.org/10.1016/j.jcis.2016.02.023

Meloni, B. P., South, S. M., Gill, D. A., Marriott, A. L., Déziel, R. A., Jacques, A., . . Knuckey, N. W. (2019). Poly-Arginine Peptides R18 and R18D Improve Functional Outcomes After Endothelin-1-Induced Stroke in the Sprague Dawley Rat. Journal of Neuropathology 85 Experimental Neurology, 78 (5), 426-435. doi:10.1093/jnen/nlz014

Meredith, P., \& Sarna, T. (2006). The physical and chemical properties of eumelanin. Pigment Cell Research, 19 (6), 572-594. doi:doi:10.1111/j.1600-0749.2006.00345.x

Mohamad, N., Loh, E. Y. X., Fauzi, M. B., Ng, M. H., \& Mohd Amin, M. C. I. (2019). In vivo evaluation of bacterial cellulose/acrylic acid wound dressing hydrogel containing keratinocytes and fibroblasts for burn wounds. Drug Delivery and Translational Research, 9 (2), 444-452. doi:10.1007/s13346-017-0475-3 
Mutschler, A., Tallet, L., Rabineau, M., Dollinger, C., Metz-Boutigue, M.-H., Schneider, F., . . Lavalle, P. (2016). Unexpected Bactericidal Activity of Poly(arginine)/Hyaluronan Nanolayered Coatings. Chemistry of Materials, 28 (23), 8700-8709. doi:10.1021/acs.chemmater.6b03872

Ng, V. W. L., Chan, J. M. W., Sardon, H., Ono, R. J., García, J. M., Yang, Y. Y., \& Hedrick, J. L. (2014). Antimicrobial hydrogels: A new weapon in the arsenal against multidrug-resistant infections.Advanced Drug Delivery Reviews, 78 , 46-62. doi:https://doi.org/10.1016/j.addr.2014.10.028

Place, E. S., George, J. H., Williams, C. K., \& Stevens, M. M. (2009). Synthetic polymer scaffolds for tissue engineering. Chemical Society Reviews, 38 (4), 1139-1151. doi:10.1039/B811392K

Poinard, B., Neo, S. Z. Y., Yeo, E. L. L., Heng, H. P. S., Neoh, K. G., \& Kah, J. C. Y. (2018). Polydopamine Nanoparticles Enhance Drug Release for Combined Photodynamic and Photothermal Therapy. ACS Applied Materials $\mathscr{E}$ Interfaces, 10 (25), 21125-21136. doi:10.1021/acsami.8b04799

Ponzio, F., Barthès, J., Bour, J., Michel, M., Bertani, P., Hemmerlé, J., . . Ball, V. (2016). Oxidant Control of Polydopamine Surface Chemistry in Acids: A Mechanism-Based Entry to Superhydrophilic-Superoleophobic Coatings. Chemistry of Materials, 28 (13), 4697-4705. doi:10.1021/acs.chemmater.6b01587

Rathna, G. V. N., Mohan Rao, D. V., \& Chatterji, P. R. (1996). Hydrogels of Gelatin-Sodium Carboxymethyl Cellulose: Synthesis and Swelling Kinetics. Journal of Macromolecular Science, Part A, 33 (9), 1199-1207. doi:10.1080/10601329608010914

Ribeiro, M., Ferraz, M. P., Monteiro, F. J., Fernandes, M. H., Beppu, M. M., Mantione, D., \& Sardon, H. (2017). Antibacterial silk fibroin/nanohydroxyapatite hydrogels with silver and gold nanoparticles for bone regeneration. Nanomedicine: Nanotechnology, Biology and Medicine, 13 (1), 231-239. doi:https://doi.org/10.1016/j.nano.2016.08.026

Sahithi, K., Swetha, M., Prabaharan, M., Moorthi, A., Saranya, N., Ramasamy, K., . . . Selvamurugan, N. (2010). Synthesis and Characterization of NanoscaleHydroxyapatite-Copper for Antimicrobial Activity Towards Bone Tissue Engineering Applications. Journal of Biomedical Nanotechnology, 6 (4), 333-339. doi:10.1166/jbn.2010.1138

Sahraro, M., Barikani, M., \& Daemi, H. (2018). Mechanical reinforcement of gellan gum polyelectrolyte hydrogels by cationic polyurethane soft nanoparticles. Carbohydrate Polymers, 187, 102-109. doi:https://doi.org/10.1016/j.carbpol.2018.01.028

Salomé Veiga, A., \& Schneider, J. P. (2013). Antimicrobial hydrogels for the treatment of infection. Peptide Science, 100 (6), 637-644. doi:doi:10.1002/bip.22412

Samberg, M. E., Loboa, E. G., Oldenburg, S. J., \& Monteiro-Riviere, N. A. (2012). Silver nanoparticles do not influence stem cell differentiation but cause minimal toxicity. Nanomedicine, 7 (8), 1197-1209. doi:10.2217/nnm.12.18

Saravanan, S., Nethala, S., Pattnaik, S., Tripathi, A., Moorthi, A., \& Selvamurugan, N. (2011). Preparation, characterization and antimicrobial activity of a bio-composite scaffold containing chitosan/nanohydroxyapatite/nano-silver for bone tissue engineering.International Journal of Biological Macromolecules, 49 (2), 188-193. doi:https://doi.org/10.1016/j.ijbiomac.2011.04.010

Sheffield, C., Meyers, K., Johnson, E., \& Rajachar, R. M. (2018). Application of Composite Hydrogels to Control Physical Properties in Tissue Engineering and Regenerative Medicine. Gels, 4 (2), 51.

Stanić, V., Janaćković, D., Dimitrijević, S., Tanasković, S. B., Mitrić, M., Pavlović, M. S., . . . Raičević, S. (2011). Synthesis of antimicrobial monophase silver-doped hydroxyapatite nanopowders for bone tissue engineering. Applied Surface Science, 257 (9), 4510-4518. doi:https://doi.org/10.1016/j.apsusc.2010.12.113

Trop, M., Novak, M., Rodl, S., Hellbom, B., Kroell, W., \& Goessler, W. (2006). Silver-Coated Dressing Acticoat Caused Raised Liver Enzymes and Argyria-like Symptoms in Burn Patient. Journal of Trauma 
and Acute Care Surgery, 60 (3), 648-652. doi:10.1097/01.ta.0000208126.22089.b6

Wang, L., Dai, W., Yang, M., Wei, X., Ma, K., Song, B., . . . Zhao, J. (2019). Cell membrane mimetic copolymer coated polydopamine nanoparticles for combined pH-sensitive drug release and near-infrared photothermal therapeutic. Colloids and Surfaces B: Biointerfaces, 176 , 1-8. doi:https://doi.org/10.1016/j.colsurfb.2018.12.057

Wei, Q., Zhang, F., Li, J., Li, B., \& Zhao, C. (2010). Oxidant-induced dopamine polymerization for multifunctional coatings. Polymer Chemistry, 1 (9), 1430-1433. doi:10.1039/C0PY00215A

Yazdimamaghani, M., Vashaee, D., Assefa, S., Walker, K. J., Madihally, S. V., Köhler, G. A., \& Tayebi, L. (2014). Hybrid Macroporous Gelatin/Bioactive-Glass/Nanosilver Scaffolds with Controlled Degradation Behavior and Antimicrobial Activity for Bone Tissue Engineering. Journal of Biomedical Nanotechnology, 10 (6), 911-931. doi:10.1166/jbn.2014.1783

Yu, H., Xu, X., Chen, X., Lu, T., Zhang, P., \& Jing, X. (2007). Preparation and antibacterial effects of PVA-PVP hydrogels containing silver nanoparticles. Journal of Applied Polymer Science, 103 (1), 125-133. doi:doi:10.1002/app.24835

Zhou, C., \& Wu, Q. (2011). A novel polyacrylamide nanocomposite hydrogel reinforced with natural chitosan nanofibers. Colloids and Surfaces B: Biointerfaces, 84 (1), 155-162. doi:https://doi.org/10.1016/j.colsurfb.2010.12.030

Zilberman, M. (2015). Drug delivery from gelatin-based systems AU - Foox, Maytal. Expert Opinion on Drug Delivery, 12 (9), 1547-1563. doi:10.1517/17425247.2015.1037272

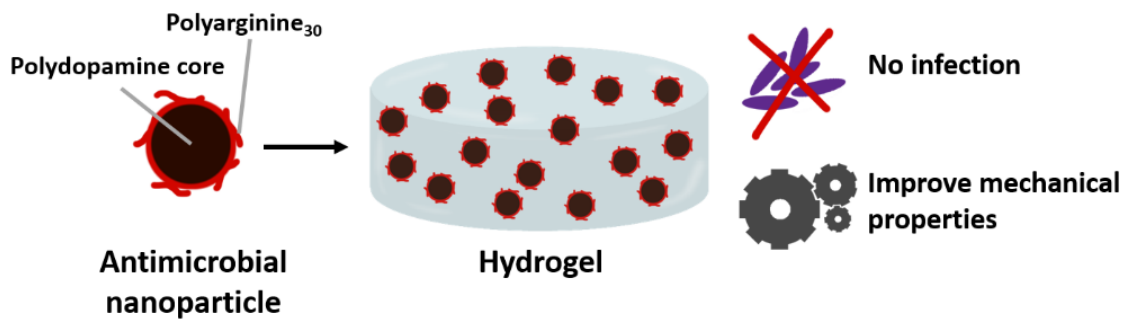

Polyarginine decorated poly-dopamine nanoparticles

TOC

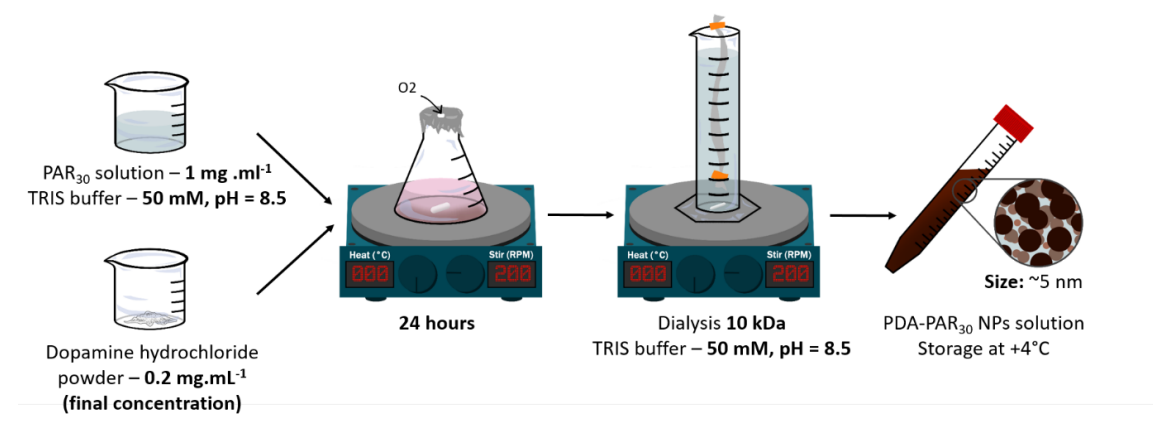


Scheme 1. Different steps to synthesize PDAPAR 30 NPs.

A)

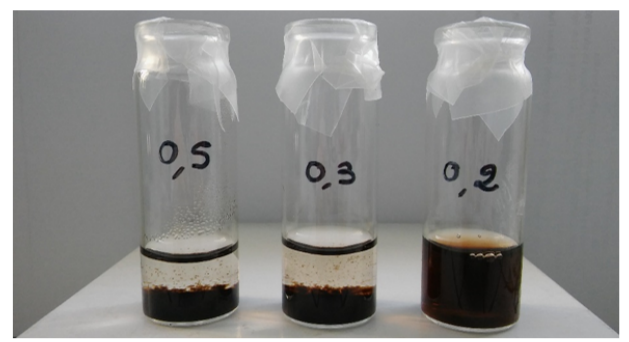

B)

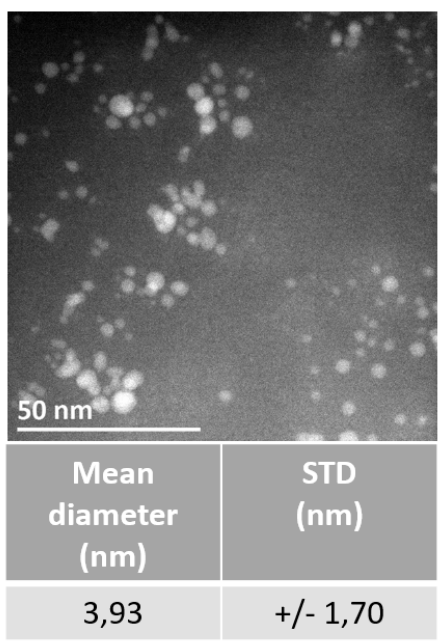

C)

\begin{tabular}{|c|c|c|c|}
\hline \multicolumn{4}{|c|}{ Zeta analysis } \\
\hline Sample name & Zeta potential $(\mathrm{mV})$ & Area $(\%)$ & $S T D(m V)$ \\
\hline PDA NPs & $-22,7$ & 100 & 8,14 \\
\hline PDA-PAR $_{30} 0.2$ NPs & 30,3 & 100 & 7,87 \\
\hline
\end{tabular}

Figure 1. A) Picture showing the stability after 2 months at $4^{\circ} \mathrm{C}$ of the different PDA-PAR 30 NPs formulations using different concentrations of dopamine hydrochloride $\left(0.5,0.3\right.$ and $\left.0.2 \mathrm{mg} . \mathrm{mL}^{-1}\right)$ with a defined concentration of polyarginine (PAR) of $1 \mathrm{mg} \cdot \mathrm{mL}^{-1}$. B). Typical image of PDA-PAR 30 0.2 NPs obtained Transmission Electron Microscope (TEM) and the subsequent size quantification results. C) Results of zeta potential measurements performed on PDA-PAR 30 0.2 NPs as compared to pure PDA particles. 


\section{A) Microbiology: S. aureus}

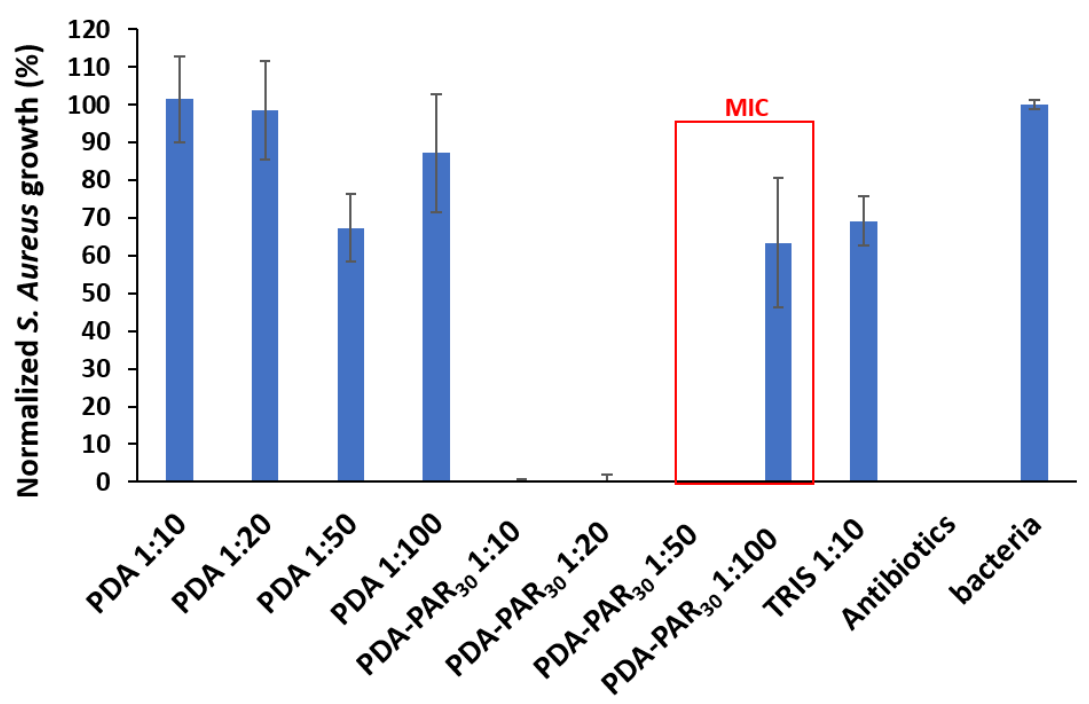

\section{B) Cytotoxicity test PDA-PAR ${ }_{30}$ NPs}

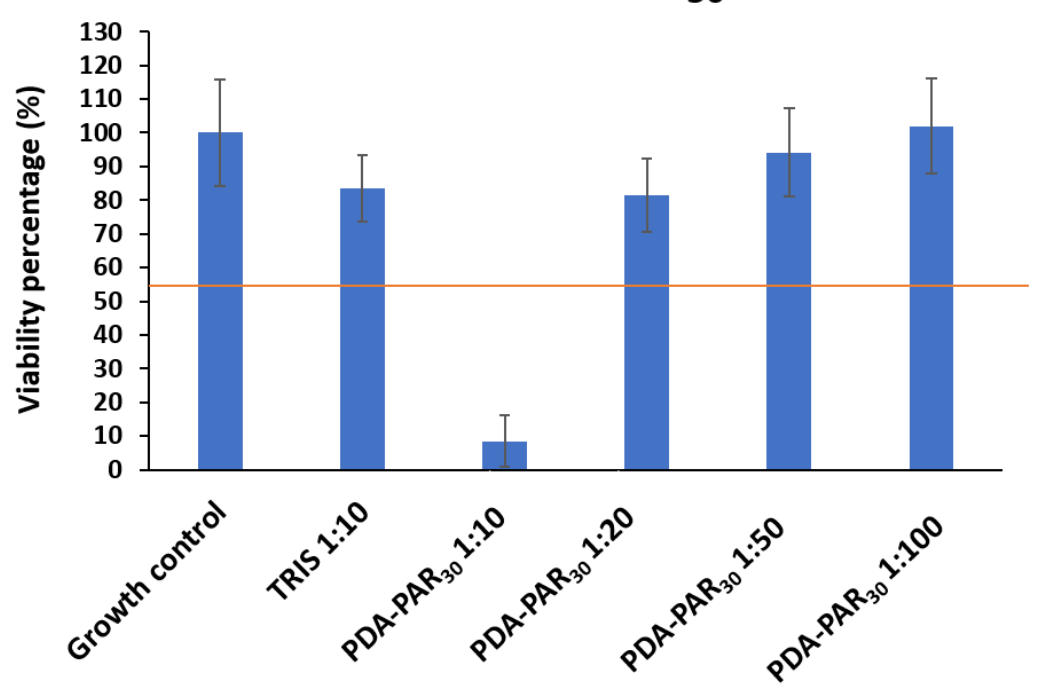

Figure 2. A) Normalized S. aureus growth in supernatant after $24 \mathrm{~h}$ in contact with different PDA-PAR 30 $0.2 \mathrm{NPs}$ at different dilution to determine the Minimum Inhibitory Concentration (MIC) ( $\mathrm{n}=3$ and error bars correspond to standard deviations). B) Cytotoxicity test of the same NPs dilutions after 24 hours of exposure with Balb 3T3 cells ( $\mathrm{n}=3$ and error bars correspond to standard deviations). 

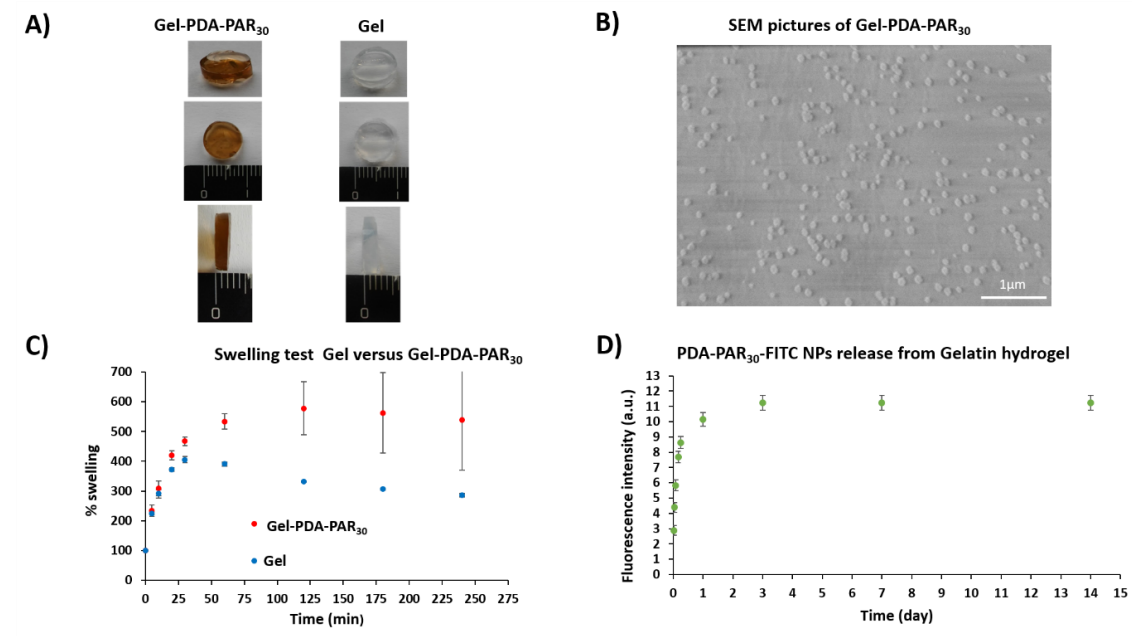

Figure 3. A) Macroscopic pictures of the resulting Gelatin NPs hydrogel composite (Gel-PDA-PAR 30 ) compared to pure Gelatin hydrogel (Gel). B) Scanning Electron Microscopy images of the Gel-PDA-PAR 30 hydrogel. C) Swelling properties of the Gel-PDA-PAR 30 hydrogel compared to pure Gelatin hydrogel at different time point in PBS at $37^{\circ} \mathrm{C}(\mathrm{n}=3$ error bars correspond to standard deviations). D) Kinetic of PDA$\mathrm{PAR}_{30}$-FITC NPs (non-diluted) release from Gelatin hydrogel at $37^{\circ} \mathrm{C}$ in PBS for 15 days. Fresh supernatant was added after each recording to perform cumulative release over time $(\mathrm{n}=3$ error bars correspond to standard deviations).

A)

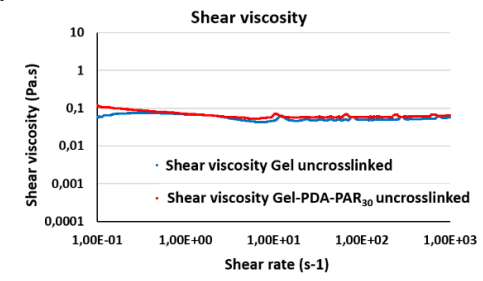

C)

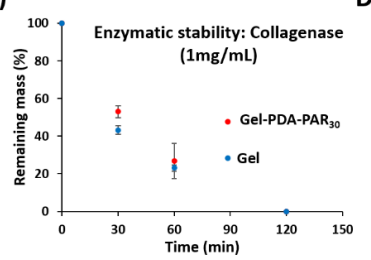

D)
B)

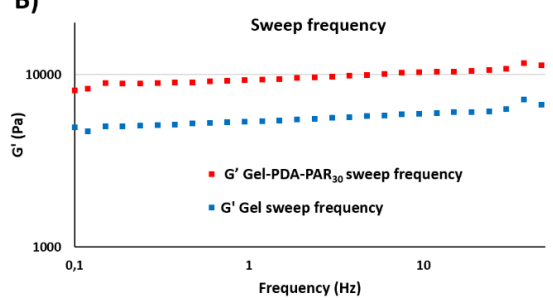

E)
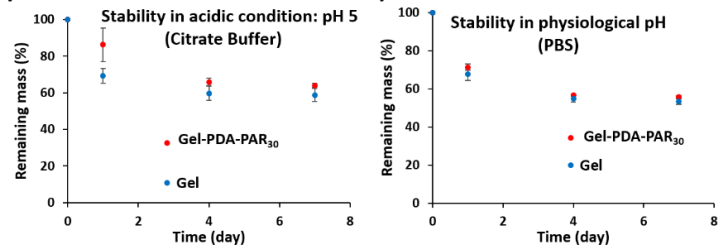

Figure 4. Rheological properties and stability of both gelatin (Gel) and Gelatin NPs composite (Gel-PDA$\mathrm{PAR}_{30}$ ) hydrogels. A) Shear viscosity as a function of shear rate for both Gel and Gel-PDA-PAR 30. B) Storage modulus $\left(G^{\prime}\right)$ as a function of frequency for both conditions tested (Gel vs Gel-PDA-PAR 30 ). Stability of the different hydrogels tested (Gel vs Gel-PDA-PAR ${ }_{30}$ ) at $37^{\circ} \mathrm{C}$ in a relevant biological medium, C) enzymatic stability in collagenase; $\mathrm{D})$ in acidic condition $(\mathrm{pH}=5)$ and in $\mathrm{E})$ physiological $\mathrm{pH}(\mathrm{pH}=7.4)$. The results are expressed as a percentage of remaining mass at different time points $(n=3$ error bars correspond to standard deviations). 

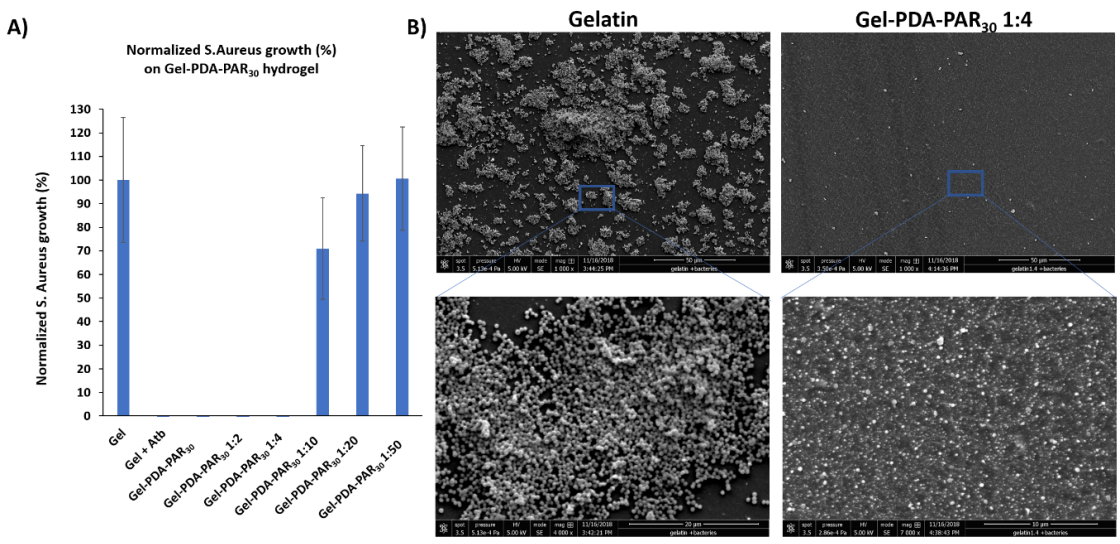

Figure 5. Normalized S. aureus growth in supernatant after $24 \mathrm{~h}$ in contact with Gelatin NPs composite hydrogel loaded with NPs (Gel-PDA-PAR 30 ) at different dilutions $(\mathrm{n}=3$ and error bars correspond to standard deviations). B) SEM images of Gelatin vs Gel-PDA-PAR 30 1:4 (NPs diluted 4 times in the hydrogel matrix) after 24 hours of exposition with S. aureus.

\section{Cytotoxicity Gel-PDA-PAR ${ }_{30}$}

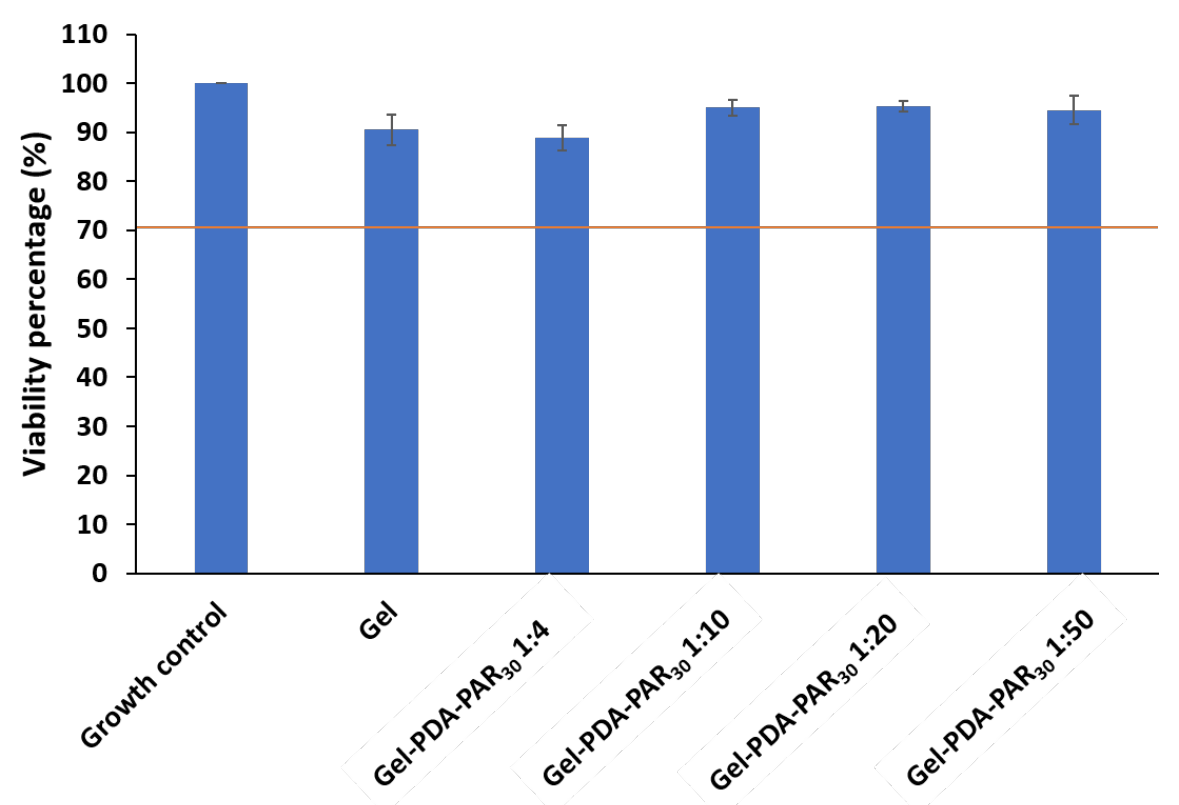

Figure 6. Cytotoxicity test with Balb 3T3 cells of Gelatin NPs composite hydrogel loaded with NPs (Gel$\mathrm{PDAPAR}_{30}$ ) at different dilutions to determine the threshold of NPs toxicity while keeping the antimicrobial activity of the resulting hydrogel ( $\mathrm{n}=3$ and error bars correspond to standard deviations). 